\title{
Triptolide is a Promising Therapeutic Approach in Treating Thyroid Cancer Based on in silico and in vitro Experiment
}

\author{
Fang Wang ${ }^{1, *}$ \\ Shu-Jing $\mathrm{An}^{2, *}$ \\ Yirong Yin ${ }^{1} * *$ \\ Juan-Juan $\mathrm{Li}^{1}$ \\ Chun-Hui Sun ${ }^{3}$ \\ Jie Lan' \\ Wen-Juan Zhao' \\ Cheng-Qian Li ${ }^{1}$
}

'Department of Endocrinology and Metabolism, The Affiliated Hospital of Qingdao University, Qingdao, 266003, People's Republic of China; ${ }^{2}$ Department of Oral Surgery, The Affiliated Hospital of Qingdao University, Qingdao, 266003, People's Republic of China; ${ }^{3}$ Department of Endocrinology, The Third People's Hospital of Qingdao Affiliated to Qingdao University, Qingdao, 266003, People's Republic of China

*These authors contributed equally to this work

\begin{abstract}
Introduction: Thyroid cancer is a familiar kind of cancer. Natural products are promising therapeutic approaches in treating thyroid cancer. Triptolide is a diterpenoid epoxide extracted from Tripterygium wilfordii. The mechanism of triptolide in the treatment of thyroid cancer has not been investigated clearly.
\end{abstract}

Methods: We evaluated triptolide targets and thyroid cancer targets with related databases. The protein-protein interaction (PPI) networks of the triptolide targets and thyroid cancer targets were constructed with Cytoscape software. Gene ontology (GO) and Kyoto Encyclopedia of Genes and Genomes (KEGG) analyses of the core PPI network were obtained. Molecular docking analysis was used to evaluated the binding of triptolide with core targets. Furthermore, apoptosis assays, real-time polymerase chain reaction (RT-PCR) and Western blotting were used to evaluate the anticancer functions of triptolide.

Results: Triptolide had 34 targets, and thyroid cancer had 210 targets. The core PPI network of merged PPI networks had 164 nodes and 4513 edges. GO and KEGG enrichment analyses showed that triptolide were related to the cell cycle, apoptosis, and inflammatory signaling pathways. Molecular docking analysis showed that triptolide directly reacted with four core targets: cyclin-dependent kinase inhibitor 1A (CDKN1A), c-JUN, RELA, and tumor protein p53 (TP53). CB-Dock analysis indicated that triptolide could stably bind to core targets. Triptolide inhibited the growth but induced apoptosis of thyroid cancer cells. Triptolide increased the mRNA expression of CDKN1A and TP53 but reduced the mRNA expression of c-JUN and RELA, as shown by RT-PCR. Triptolide increased the protein levels of CDKN1A and phospho-p53 but reduced those of phospho-c-JUN and phospho-NF- $\kappa$ B p65, as shown by Western blotting.

Discussion: We considered that triptolide could treat thyroid cancer by inhibiting cell proliferation, inducing apoptosis and inhibiting inflammatory pathways such as the NF- $\mathrm{BB}$ and MAPK signaling pathways. CDKN1A, c-JUN, RELA, and TP53 were involved in the antithyroid cancer mechanism of triptolide.

Keywords: triptolide, thyroid cancer, molecular docking, apoptosis, inflammation

\section{Introduction}

Thyroid cancer is a type of cancer that develops in the thyroid gland. ${ }^{1}$ According to the newest thyroid cancer epidemiology, the incidence of thyroid cancer was 586,202 cases and number of new deaths was 43,646 in 2020, worldwide. ${ }^{2}$ Women aged between 35 and 65 are commonly affected by thyroid cancer. The causes of thyroid cancer are unclear, although environmental and genetic factors contribute to thyroid cancer. The subtypes of thyroid cancer according to histopathological characteristics include
Cheng-Qian Li

Department of Endocrinology and Metabolism, The Affiliated Hospital of Qingdao University, No. 16 Jiangsu Road, Qingdao, 266003, Shandong Province,

People's Republic of China

Email18661807103@I63.com;

lichengqian5213@qdu.edu.cn 
papillary thyroid cancer, follicular thyroid cancer, medullary thyroid cancer, etc. ${ }^{3}$ Papillary thyroid cancer is the most common type of thyroid cancer. In addition, papillary thyroid cancer has the best overall prognosis among the subtypes of thyroid cancer. ${ }^{4}$ The symptoms of thyroid cancer are atypical in the early stage, and the first symptom might be a nodule in the thyroid region. ${ }^{5}$ The other symptoms of thyroid cancer include hoarseness, difficulty swallowing, neck pain, and swollen lymph nodes. The treatment methods include radiotherapy, surgery, thermotherapy, thyroid hormone therapy, targeted drug therapy, etc. However, treatments for thyroid cancer have some adverse effects. Therefore, it is important to investigate new therapeutic methods for thyroid cancer.

Triptolide is extracted from thunder god vine, Tripterygium wilfordii. It has been demonstrated to be useful in treating various types of cancer. ${ }^{6}$ A previous study found that triptolide was useful against lung, liver, and breast cancer. ${ }^{7,8}$ Triptolide was found to suppress lung cancer invasion, migration, and metastasis. ${ }^{9}$ Triptolide was also found to inhibit breast cancer cell proliferation and invasion. However, the mechanism of triptolide in the treatment of thyroid cancer has not been clarified.

In this study, we used network pharmacology and molecular docking analyses to investigate the antithyroid cancer function of triptolide. First, we evaluated triptolide targets and thyroid cancer targets. Second, we generated the protein-protein interaction (PPI) networks of the triptolide targets and thyroid cancer targets. Cytoscape software was used to merge the two PPI networks. Then, Gene ontology (GO) and Kyoto Encyclopedia of Genes and Genomes (KEGG) analyses of the core PPI network were obtained. Via molecular docking analysis, we evaluated the binding of triptolide with the four core targets. Finally, we demonstrated the function of triptolide in treating thyroid cancer in vitro. Apoptosis assays, real-time polymerase chain reaction (RT-PCR) and Western blotting were used to evaluate the anticancer functions of triptolide. We aimed to reveal the mechanism of triptolide in treating thyroid cancer in this study. Our research could provide theoretical and experimental findings to support future studies.

\section{Methods}

\section{Evaluation of Triptolide Targets and Thyroid Cancer Targets}

In this study, we used traditional Chinese medicine systems pharmacology (TCMSP) database to evaluate the targets of triptolide. The TCMSP database is a useful pharmacological database containing chemical, drug and target networks. In addition, we used the Genetic Association Database (GAD), Online Mendelian Inheritance in Man (OMIM), Therapeutic Target Database (TTD), and Pharmacogenomics Knowledge Base (PharmGKB) databases to investigate the targets of thyroid cancer. The GAD database provides information about the relationships between diseases and genes. The OMIM database also contains data on diseases and genes. The TTD database provides information about proteins, nucleic acids and related targets. The PharmGKB database includes information on the effects of genetic variation on drug responses.

\section{PPI Network Construction}

In this study, a PPI network was constructed with the BisoGenet plugin. The databases providing data for the PPI network included Database for Interacting Proteins (DIP), Biological General Repository for Interaction Datasets (BioGRID), IntAct, Biomolecular Interaction Network Database (BIND), Human Protein Reference Database (HPRD), and Molecular INTeraction Database (MINT). Then, Cytoscape software was used to visualize the PPI network. Cytoscape was also used to merge the triptolide PPI network and the thyroid cancer PPI network. Furthermore, topological features were evaluated by parameters including the eigenvector centrality (EC), closeness centrality (CC), local average connectivity centrality (LAC), betweenness centrality (BC), degree centrality (DC), and neighbourhood centrality (NC). The CytoNCA plugin was used in the topological feature analysis.

\section{GO and KEGG Analyses}

In this study, GO and KEGG analyses were conducted with the DAVID database. GO analysis included the biological process (BP), molecular function (MF), and cellular component (CC) categories. KEGG analysis revealed correlated genes, drugs, molecular pathways, etc.

\section{Molecular Docking Analysis}

We used CB-Dock and AutoDock to investigate the physical interactions between triptolide and the target proteins. CB-Dock can analyze the center, size, and binding sites. CB-Dock uses a cavity detection approach. In CB-Dock, ligands and receptors are visualized as space-filling representations and cartoons. The colors of the receptors and ligands are determined by the chains and elements. Discovery Studio contains tools for structure design, 
ligand design, and simulation. The ligand design tools include library optimization and molecular library construction. The simulations include quantum and molecular mechanics simulations. AutoDock is a suite of automated docking tools to predict the hydrogen bonds of small molecules. These two are powerful models to explore the relationships between drugs and proteins.

\section{Cells and Reagents}

The thyroid cancer cell line TPC-1 was selected for this study. TPC-1 cells were purchased from Bena Culture Collection (Henan, China). RPMI 1640 medium (Corning, 10-040-cv) was supplemented with $10 \%$ fetal bovine serum (FBS) (Gibco, 10099141), Penicillin-Streptomycin solution (Corning, 30-001-CI). TPC-1 cells were maintained under humidified conditions with $5 \% \mathrm{CO} 2$ at $37^{\circ} \mathrm{C}$. Triptolide was purchased from Merck (T3652). Cell apoptosis kits containing propidium iodide (PI) and Annexin V- Fluorescein isothiocyanate (FITC) were purchased from Abcam (ab14085). The anti- cyclin-dependent kinase inhibitor 1A (CDKN1A) primary antibody was purchased from Sigma-Aldrich (SAB5700742). Anti-phospho-c-JUN (CST, 3270s), antiphospho-p53 (CST, 2521s), anti-phospho-NF-кB p65 (CST, 3031s), and anti- glyceraldehyde 3-phosphate dehydrogenase (GAPDH) (CST, 5174s) antibodies were purchased from Cell Signaling Technology. Goat anti-rabbit secondary antibody was purchased from Abcam (ab6721).

\section{Treatment Protocol of Cells}

In the subculture of cells, we viewed cultures using an inverted microscope to assess the degree of confluency. After washing the culture flask with PBS, 0.25\% Trypsin/ Ethylenediaminetetraacetic acid (EDTA) (Gibco, 2186970) was pipetted. After pouring the excess trypsin, the culture flask was returned to the incubator and leave for $5 \mathrm{~min}$ utes. Then, we resuspend the cells in a small volume of fresh serum-containing medium to inactivate the trypsin. Cells were counted and required number of cells were transferred in new labelled culture flask.

In the apoptosis assay, cells were cultured in 6-well plates with $1.2 \times 10^{5}$ cells in every well. After $24 \mathrm{~h}$ culture, PBS was used to wash the plate and different concentrations of triptolide were added into labelled wells. After $24 \mathrm{~h}$ treatment, $0.25 \%$ Trypsin without EDTA (Gibco, 2195440) was used to digest the cells. Then, cells were collected to conduct the apoptosis assay. In Western blot and RT-PCR analysis, cells were cultured in 6-well plates with $4 \times 10^{5}$ cells in every well. After $24 \mathrm{~h}$ culture, PBS was used to wash the plate and different concentrations of triptolide were added into labelled wells. After $24 \mathrm{~h}$ treatments, cells were collected to conduct the experiments.

\section{Cell Proliferation Assay}

In this study, we used an MTT assay (Biorigin, BN20390) to evaluate the proliferation of thyroid cancer cells treated with triptolide. TPC-1 cells were treated with various concentrations of triptolide in 96-well plates. The plates were incubated with MTT solution after $48 \mathrm{~h}$ of incubation alone. Finally, the absorbance of each plate well was evaluated with a microplate reader.

\section{Western Blot Analysis}

After treatment with triptolide for $24 \mathrm{~h}$, cells were lysed with RIPA buffer (Beyotime, P0013B) containing phenylmethylsulfonyl fluoride (PMSF) for $20 \mathrm{~min}$. The proteins were separated at $120 \mathrm{~V}$ for $80 \mathrm{~min}$. After transfer at 120 $\mathrm{V}$ for $150 \mathrm{~min}$, the membrane was incubated with $5 \%$ milk for $2 \mathrm{~h}$. Then, the membrane was incubated with primary antibodies overnight at $4{ }^{\circ} \mathrm{C}$. After washing with phosphate buffered saline with Tween 20 (PBST) 3 times, the membrane was incubated with the secondary antibody. Finally, immunoreactions on the membrane were detected.

\section{RT-PCR Analysis}

SYBR Green Master Mix was used in this experiment. The primers were as follows: CDKN1A (forward: 5'gggctgggagtagttgtctt-3', reverse: 5'- attgtgggaggagctgtgaa3'), c-JUN (forward: 5'- tttcaggaggctggaggaag-3', reverse: 5'- ctgccaccaattcctgcttt-3'), RELA (forward: 5'- agaagcaggctggaggtaag-3', reverse: 5'- gccatggttgagcaaggaaa-3'), tumor protein p53 (TP53) (forward: 5'- tggecatctacaagcagtca-3', reverse: 5'- ggtacagtcagagccaacct-3'), and GAPDH (forward: 5'- ccaaggagtaagacccctgg-3', reverse: 5'- tggttgagcacagtctt-3'). TRIzol (Invitrogen, 15596018) was used to acquire total RNA. Then, ReverTra Ace qPCR RT Master Mix (Toyobo, FSQ-201) was conducted to reverse transcribe $1 \mu \mathrm{g}$ RNA to cDNA. SYBR Green RTPCR Master Mix (Toyobo, QPK-201) was used to perform RT-PCR. 3-step cycle was used in cycling condition of RealTime PCR System with 40 cycles. $2^{-\Delta \Delta \text { Ct }}$ method was conducted to evaluate relative expression. $\beta$-actin expression was used to normalize gene expression.

\section{Apoptosis Assay}

TPC-1 thyroid cancer cells were tested with a cell apoptosis kit. After centrifugation, cells were resuspended in $500 \mu \mathrm{L}$ of 
$1 \mathrm{X}$ Annexin $\mathrm{V}$ binding buffer. Then, we added $5 \mu \mathrm{L}$ of Annexin V-FITC and $5 \mu \mathrm{L}$ of propidium iodide. Furthermore, we incubated the cells at room temperature for $5 \mathrm{~min}$ in the dark. Finally, we analyzed the cells by flow cytometry.

\section{Statistical Analysis}

GraphPad Prism was used to analyze the results. Student's two-tailed $t$-test was used to evaluate statistical significance. $\mathrm{P}<0.05$ was considered statistically significant.

\section{Results}

\section{Evaluation of Triptolide Targets and Thyroid Cancer Targets}

There were 34 targets of triptolide in the TCMSP dataset. The triptolide targets included B-cell lymphoma $2(\mathrm{Bcl} 2)$, CDKN1A, C-X-C Motif Chemokine Ligand 8 (CXCL8), c-JUN, Mitogen-Activated Protein Kinase 8 (MAPK8), etc. (Figure 1A). There were 210 targets of thyroid cancer in the GAD, OMIM, TTD, and PharmGKB databases. The thyroid cancer targets included A-Kinase Anchoring Protein 9 (AKAP9), AKT1, Cholecystokinin B Receptor (CCKBR), CD80, Cyclin Dependent Kinase Inhibitor 1B (CDKN1B), 3-Hydroxy-3-Methylglutaryl-CoA Reductase (HMGCR), Janus Kinase 1 (JAK1), etc. (Figure 1B).

\section{Construction of PPI Networks}

In this study, we constructed PPI networks of triptolide and thyroid cancer targets. First, we constructed the triptolide target PPI network. The triptolide target PPI network had 2551 nodes and 52,255 edges (Figure 2A). The thyroid cancer target PPI network had 6694 nodes and 164,435 edges (Figure 2B). Then, the triptolide PPI network and thyroid cancer PPI network were merged. The merged PPI network contained 2058 nodes and 47,470 edges (Figure 2C). The filtering parameters included DC $>60, \quad \mathrm{BC}>0.000837712025133824, \mathrm{CC}>0.53135313531$ 3531, EC $>0.0340744964778423, \quad$ LAC $>18.0862068965$ 517 , and $\mathrm{NC}>19.7636224602856$. Finally, the core PPI network included 164 nodes and 4513 edges (Figure 2D).

\section{Functional Enrichment Analysis of Triptolide in Treating Thyroid Cancer}

We conducted GO and KEGG enrichment analyses to discover the mechanism of triptolide in treating thyroid cancer. GO analysis showed the biological processes related to the treatment of thyroid cancer by triptolide; these biological process terms included apoptotic process, cell cycle arrest, NF-kappa B transcription factor activity, stress-activated mitogen-activated protein kinases (MAPK) cascade, G2/M transition of mitotic
A

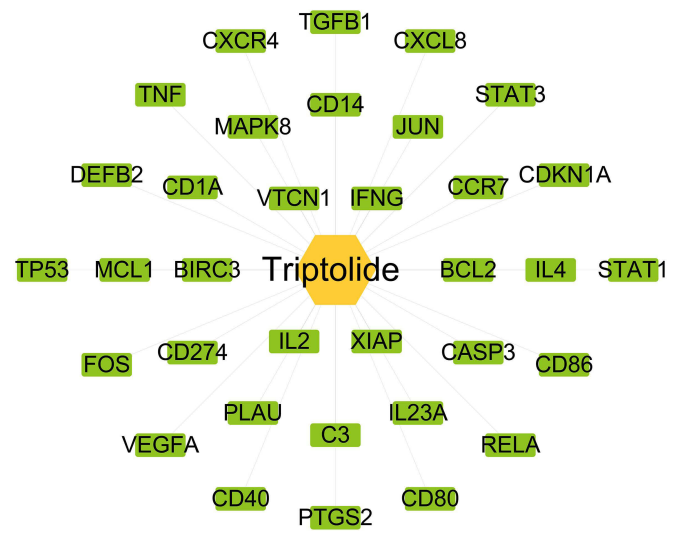

Triptolide targets

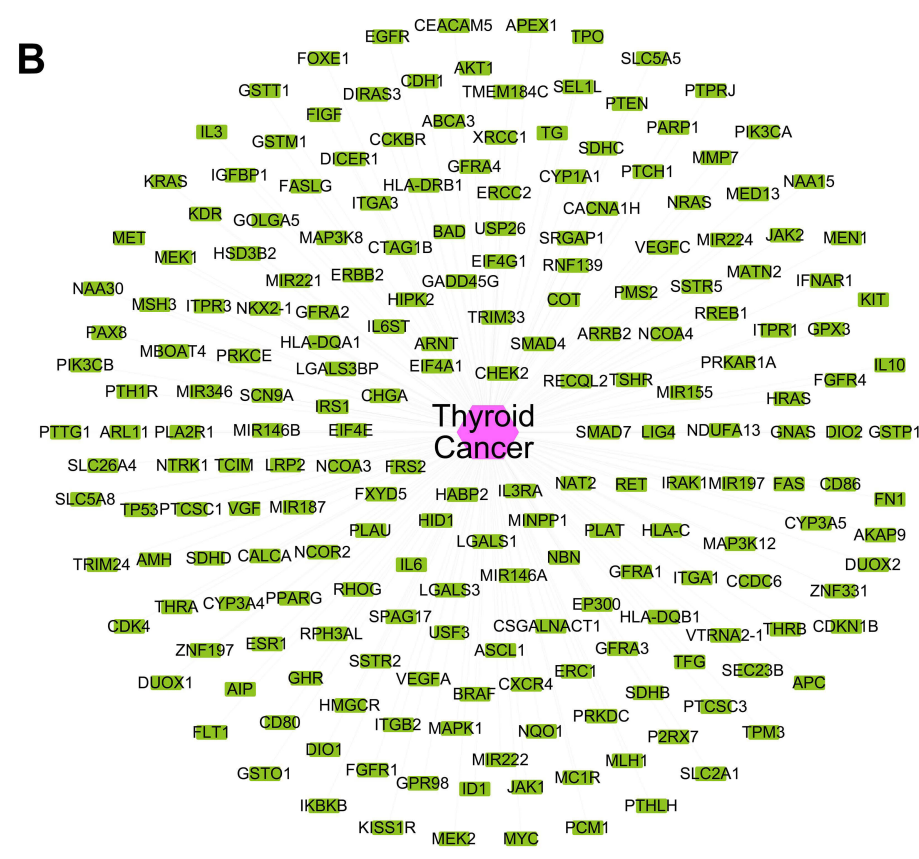

Thyroid cancer targets

Figure I Evaluation of triptolide targets and thyroid cancer targets. (A) The potential targets of triptolide. (B) The potential targets of thyroid cancer. 

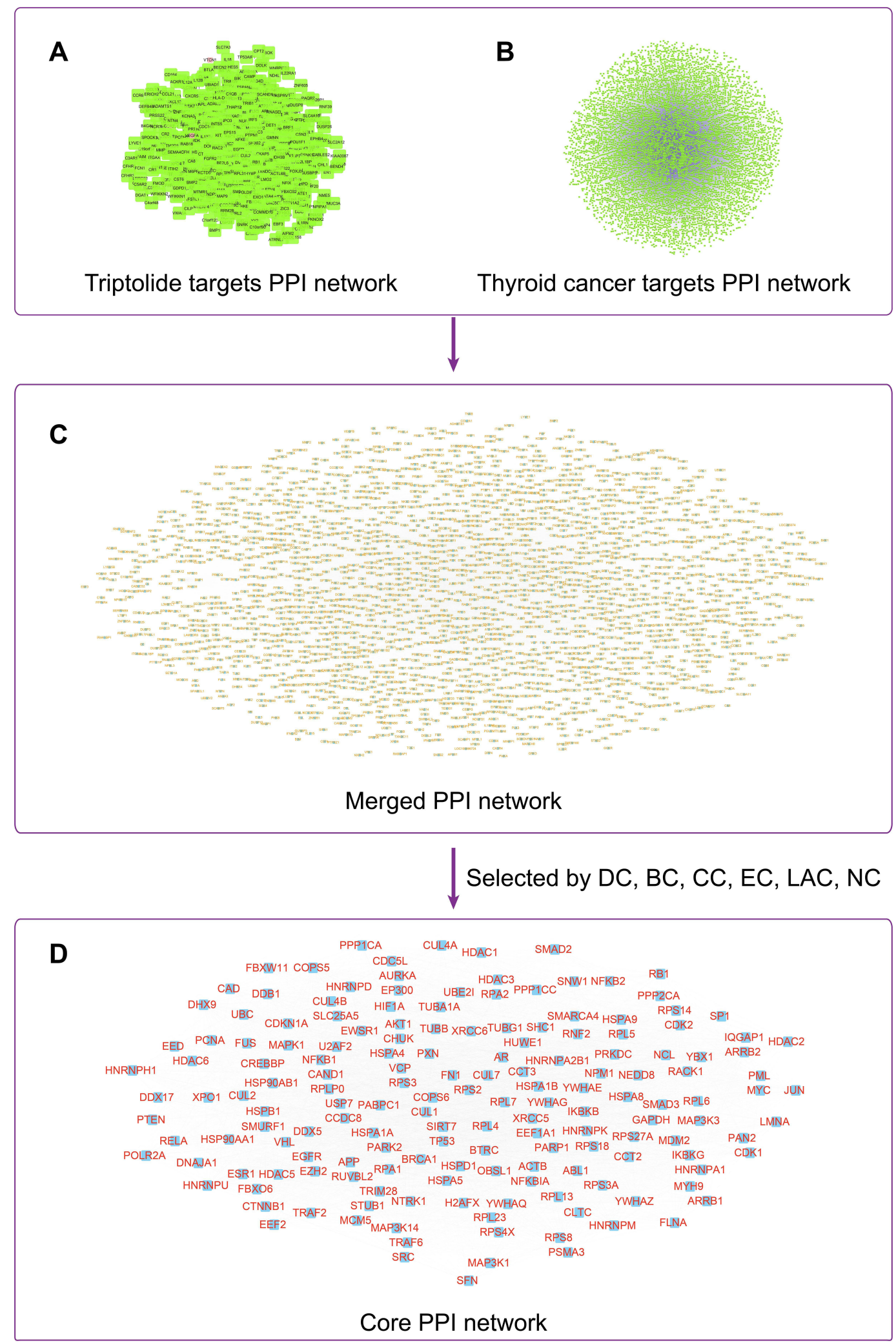

Figure 2 Construction of PPI networks. (A) Triptolide target PPI network. (B) Thyroid cancer target PPI network. (C) Merged PPI network. (D) Core PPI network. 

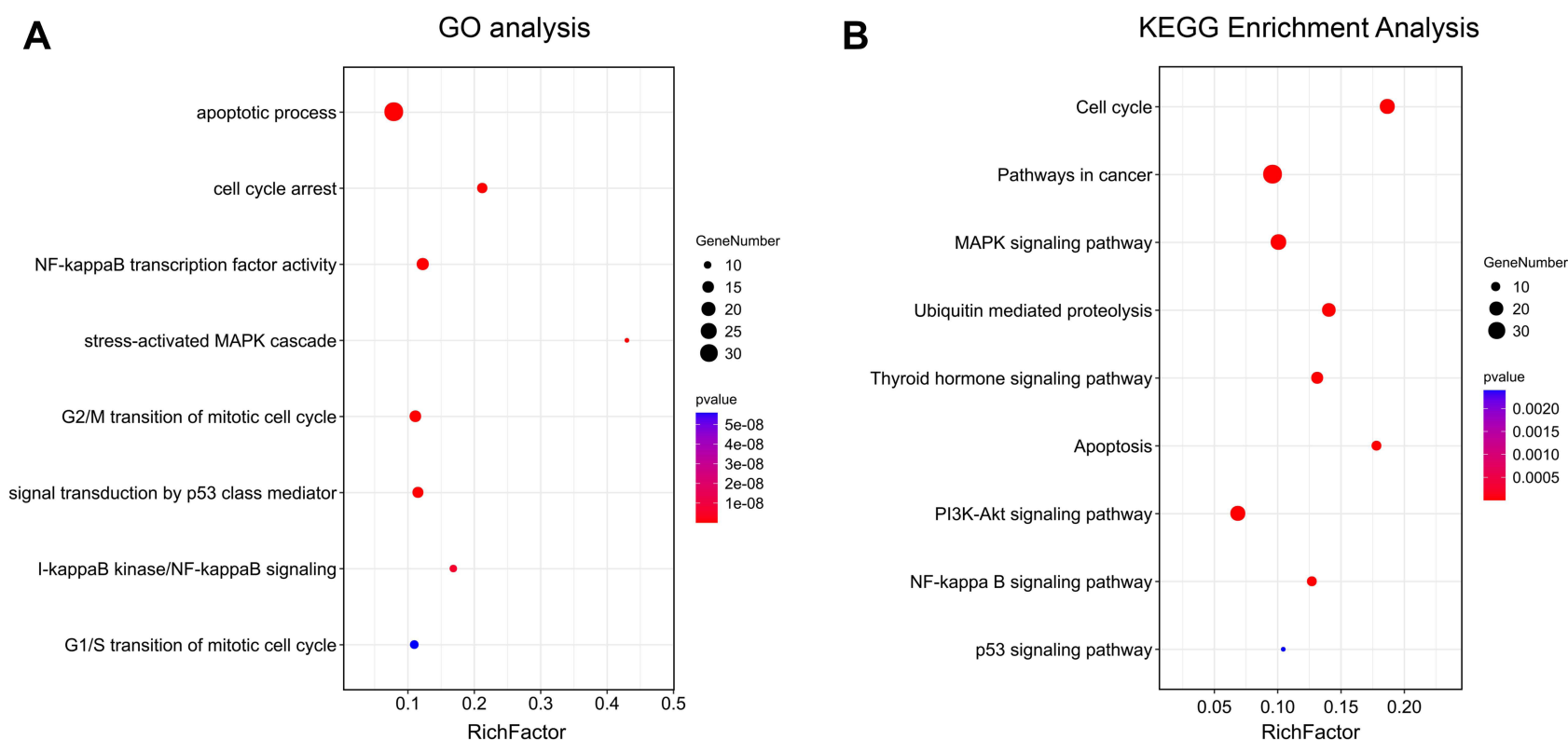

Figure 3 Functional enrichment analysis. (A) GO biological process (BP) analysis. Enrichment scores are shown on the $X$ axis, while $G O$ terms enriched with target genes are shown on the $Y$ axis. (B) The related pathways of triptolide in treating thyroid cancer. The enrichment scores are listed on the $X$ axis, while target gene-enriched items are shown on the $Y$ axis.

cell cycle, signal transduction by 553 class mediator, I-kappaB kinase/NF-kappa B signaling, G1/S transition of mitotic cell cycle, etc. (Figure 3A). KEGG analysis showed that the pathways related to the treatment of thyroid cancer by triptolide included the cell cycle, pathways in cancer, MAPK signaling pathway, ubiquitin-mediated proteolysis, thyroid hormone signaling pathway, apoptosis, PI3K-Akt signaling pathway, NFkappa B signaling pathway, and p53 signaling pathway (Figure 3B).

\section{Molecular Docking Analysis}

We used CB-Dock to evaluate the interactions between triptolide and the core targets. We combined the triptolide targets and the core PPI network to obtain the core targets (Figure 4). The four core targets were CDKN1A, c-JUN, RELA, and TP53. Then, we analyzed the Vina scores for the binding of triptolide with CDKN1A, c-JUN, RELA, and TP53 (Figure 5). The results showed that the lowest Vina score for the binding of triptolide to CDKN1A was $-6.9 \mathrm{kcal} / \mathrm{mol}$. The lowest Vina score for the binding of triptolide to c-JUN was $-5.7 \mathrm{kcal} / \mathrm{mol}$. The lowest Vina score for the binding of triptolide to RELA was $-6.9 \mathrm{kcal} / \mathrm{mol}$. The lowest Vina score for the binding of triptolide to TP42 was $-8.9 \mathrm{kcal} /$ mol. These results indicated that triptolide can bind stably to the core targets.

\section{Triptolide Suppressed the Growth of Thyroid Cancer Cells}

The thyroid cancer cell line TPC-1 was used to investigate the antithyroid cancer function of triptolide. We used various concentrations of triptolide (50 $\mathrm{nM}$ to $200 \mathrm{nM}$ ) to treat TPC-1 cells. The results of the MTT assay showed that triptolide inhibited TPC-1 cell proliferation in a dosedependent manner (Figure 6). Triptolide inhibited thyroid cancer cell growth.

\section{Triptolide Regulated the mRNA Expression of the Core Targets}

RT-PCR analysis was used to measure the effects of triptolide on the mRNA expression of the core targets. Triptolide increased the mRNA expression of CDKN1A and TP53 in a dose-dependent manner $(50 \mathrm{nM}, 100 \mathrm{nM}$, $200 \mathrm{nM}$ ). Triptolide suppressed the expression of c-JUN and RELA in a dose-dependent manner $(50 \mathrm{nM}, 100 \mathrm{nM}$, $200 \mathrm{nM}$ ) (Figure 7).

\section{Triptolide Regulated the Protein Levels of the Core Targets in TPC-I Cells}

We used Western blotting to evaluate the function of triptolide in regulating the core targets. Triptolide increased the protein levels of CDKN1A and phospho$\mathrm{p} 53$ in a dose-dependent manner $(50 \mathrm{nM}, 100 \mathrm{nM}$, 


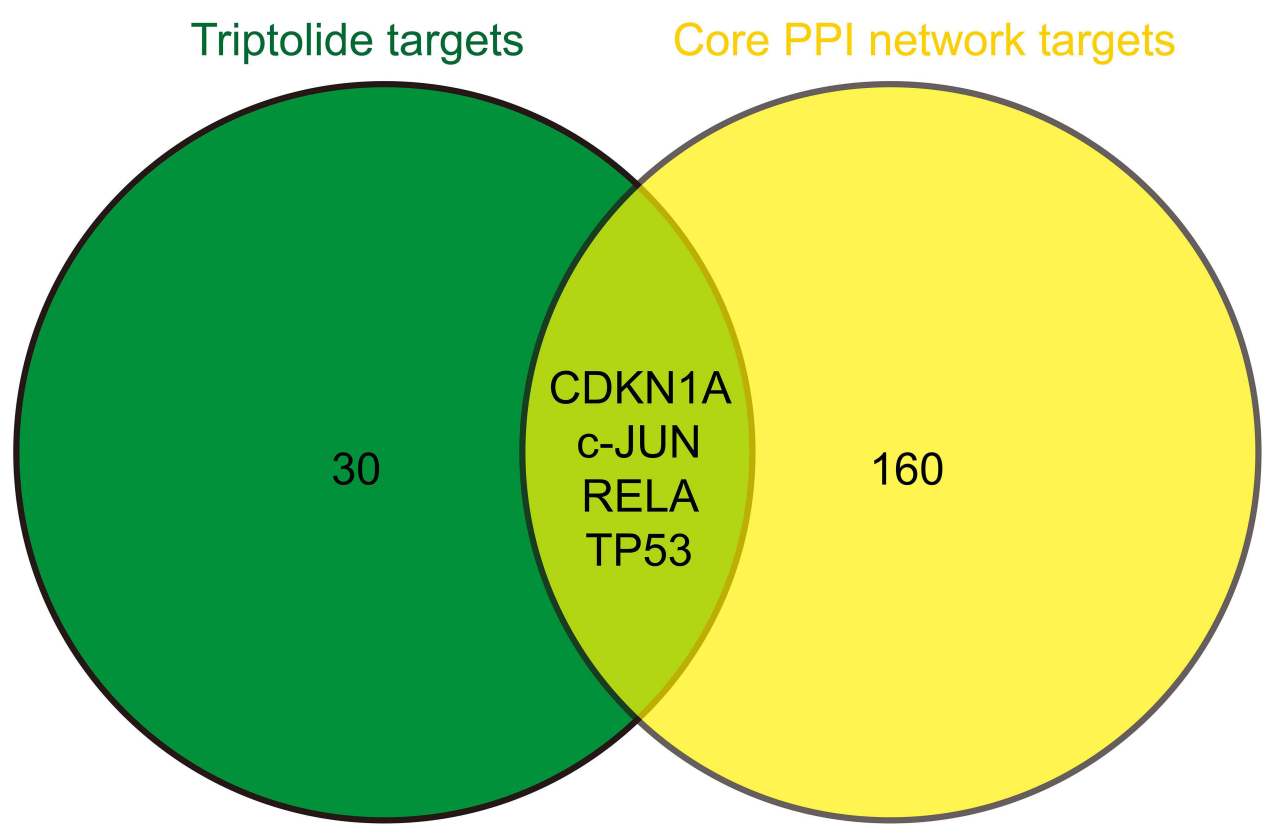

Figure 4 Venn diagram of triptolide targets and the core PPI network.

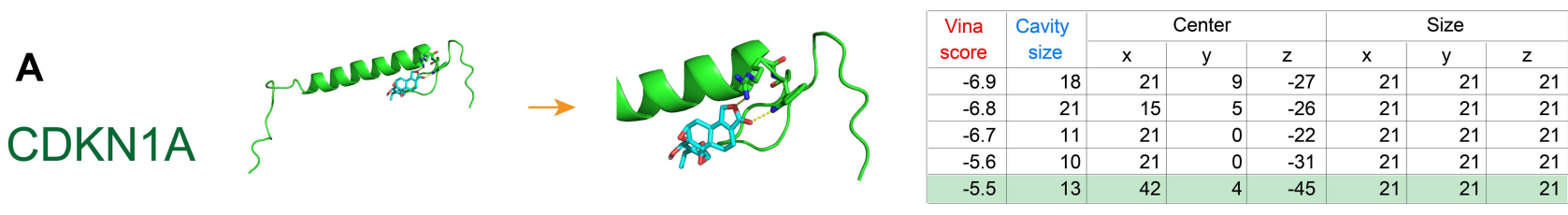

B

C-JUN

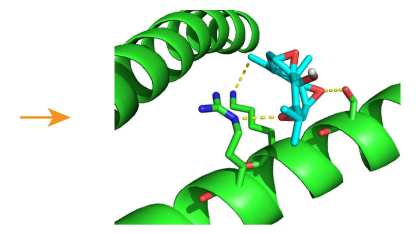

\begin{tabular}{|r|r|r|r|r|r|r|r|}
\hline \multirow{2}{*}{$\begin{array}{c}\text { Vina } \\
\text { score }\end{array}$} & Cavity & \multicolumn{3}{|c|}{ Center } & \multicolumn{3}{|c|}{ Size } \\
\hline & size & $\mathrm{x}$ & \multicolumn{1}{|c|}{$\mathrm{y}$} & \multicolumn{1}{|c|}{$\mathrm{z}$} & $\mathrm{x}$ & $\mathrm{y}$ & $\mathrm{z}$ \\
\hline-5.7 & 24 & 7 & -1 & 16 & 21 & 21 & 21 \\
\hline-5.7 & 22 & 15 & 0 & 17 & 21 & 21 & 21 \\
\hline-5.2 & 33 & 15 & 8 & 19 & 21 & 21 & 21 \\
\hline-5.1 & 24 & 13 & 4 & 53 & 21 & 21 & 21 \\
\hline-5 & 21 & 7 & -3 & 52 & 21 & 21 & 21 \\
\hline
\end{tabular}

C

RELA

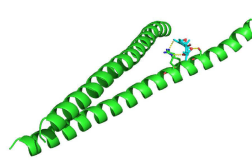

\begin{tabular}{|c|c|c|c|c|c|c|c|}
\hline \multirow{2}{*}{$\begin{array}{l}\text { Vina } \\
\text { score }\end{array}$} & \multirow{2}{*}{$\begin{array}{c}\text { Cavity } \\
\text { size }\end{array}$} & \multicolumn{3}{|c|}{ Center } & \multicolumn{3}{|c|}{ Size } \\
\hline & & $x$ & $y$ & $z$ & $x$ & $y$ & $z$ \\
\hline-7.5 & 304 & -12 & 57 & 39 & 21 & 21 & 21 \\
\hline-6.9 & 291 & -4 & 37 & 19 & 21 & 21 & 21 \\
\hline-6.7 & 802 & -5 & 51 & 12 & 21 & 21 & 21 \\
\hline-6.6 & 326 & 3 & 61 & 30 & 21 & 21 & 21 \\
\hline-6.6 & 252 & 8 & 46 & 30 & 21 & 21 & 21 \\
\hline
\end{tabular}
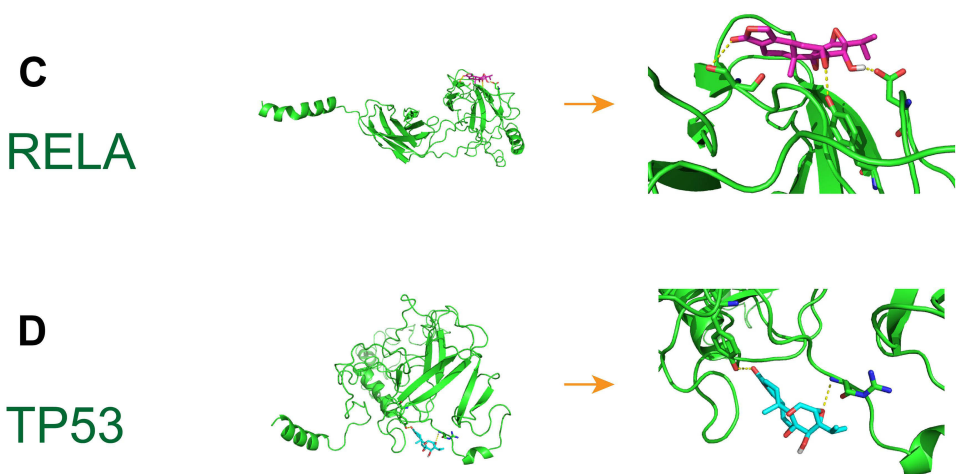

\begin{tabular}{|r|r|r|r|r|r|r|r|}
\hline Vina & Cavity & \multicolumn{3}{|c|}{ Center } & \multicolumn{3}{|c|}{ Size } \\
\cline { 3 - 8 } score & size & \multicolumn{1}{|c|}{$\mathrm{x}$} & \multicolumn{1}{c|}{$\mathrm{y}$} & \multicolumn{1}{c|}{$\mathrm{z}$} & \multicolumn{1}{c|}{$\mathrm{x}$} & \multicolumn{1}{c|}{$\mathrm{y}$} & \multicolumn{1}{c|}{} \\
\hline-8.9 & 1320 & 235 & 135 & 231 & 21 & 21 & 21 \\
\hline-7.8 & 644 & 218 & 142 & 222 & 21 & 21 & 21 \\
\hline-7.1 & 1335 & 217 & 143 & 231 & 21 & 27 & 21 \\
\hline-6.3 & 974 & 237 & 145 & 222 & 21 & 21 & 21 \\
\hline-5.6 & 2123 & 227 & 158 & 240 & 31 & 21 & 30 \\
\hline
\end{tabular}

Figure 5 Molecular docking analysis. (A) The binding affinity of triptolide for CDKNIA (lowest Vina score $=-6.9$, cavity size $=18$ ). $(\mathbf{B})$ The binding affinity of triptolide for c-JUN (lowest Vina score $=-5.7$, cavity size $=24)$. (C) The binding affinity of triptolide for RELA (lowest Vina score $=-7.5$, cavity size $=304$ ). $($ D) The binding affinity of triptolide for TP53 (lowest Vina score $=-8.9$, cavity size $=1320$ ). 


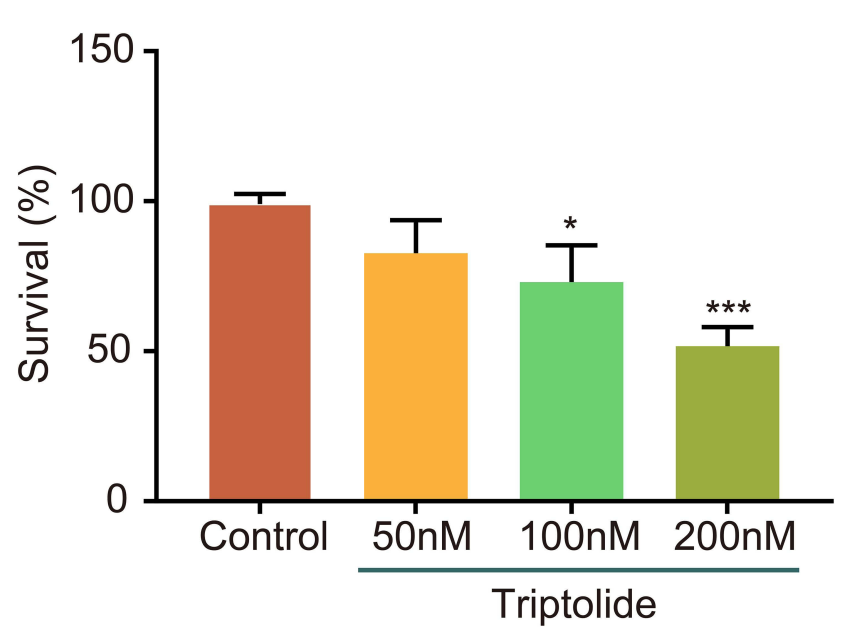

Figure 6 MTT assay of triptolide-treated TPC-I cells. The curve shows that triptolide inhibited TPC-I cell growth in a dose-dependent manner. $* P<0.05$, $* * * P<0.001$.

$200 \mathrm{nM})$. In addition, triptolide decreased the levels of phospho-c-JUN and phospho-NF-кB p65 in a dosedependent manner (50 nM, 100 nM, 200 nM) (Figure 8).
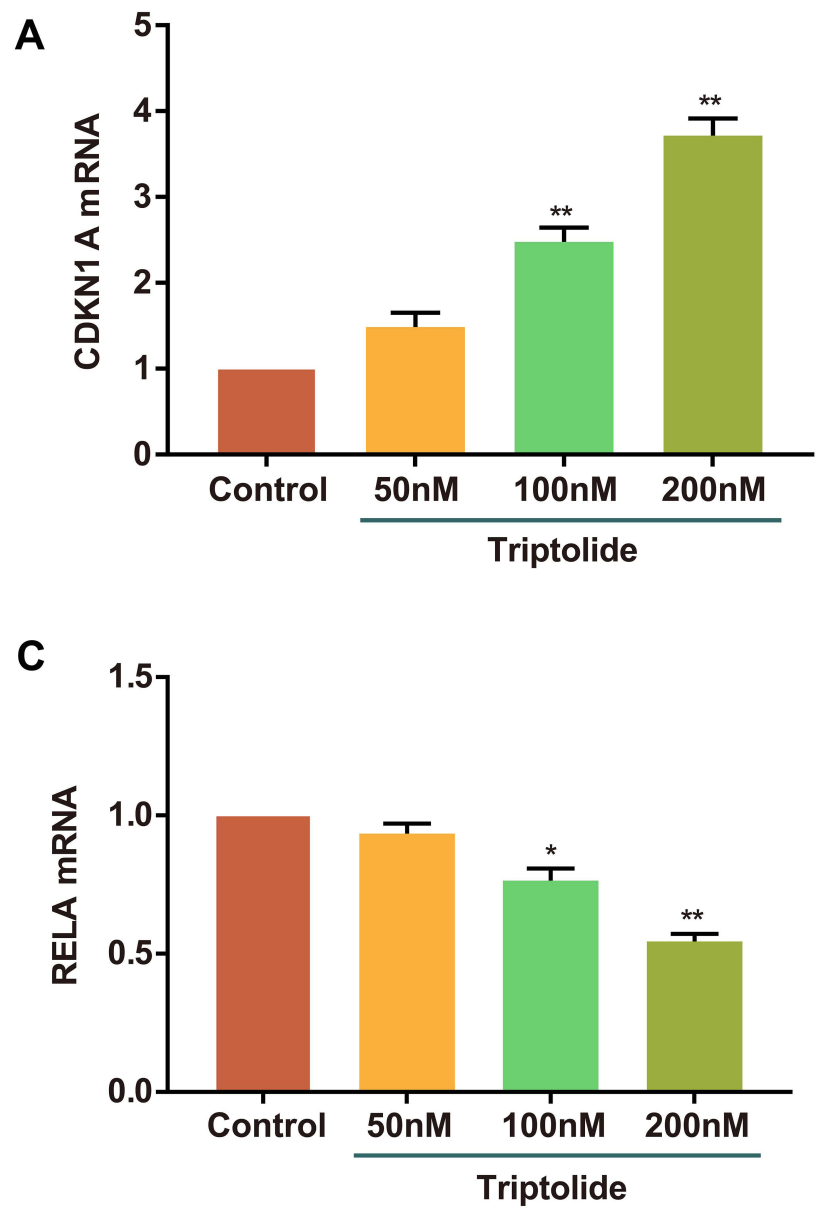

Triptolide Induced the Apoptosis of Thyroid Cancer Cells

TPC-1 cells were incubated with different concentrations of triptolide (50 nM, $100 \mathrm{nM}, 200 \mathrm{nM}$ ) for $24 \mathrm{~h}$. We used a cell apoptosis detection kit to evaluate the apoptosis of TPC-1 cells. After incubation, TPC-1 cells were subjected to Annexin V-FITC and PI staining with the kit reagents. We found that triptolide induced thyroid cancer cell apoptosis in a dose-dependent manner (50 nM, $100 \mathrm{nM}, 200$ $\mathrm{nM}$ ) (Figure 9).

\section{Discussion}

In this study, we evaluated the function of triptolide in treating thyroid cancer with in silico and in vitro experiment. In network pharmacology, we found that inflammatory pathways, cell cycle and apoptosis were involved in triptolide treating thyroid cancer. Molecular docking analysis showed that triptolide directly interacted with core targets. In vitro experiment showed triptolide inhibited the

B

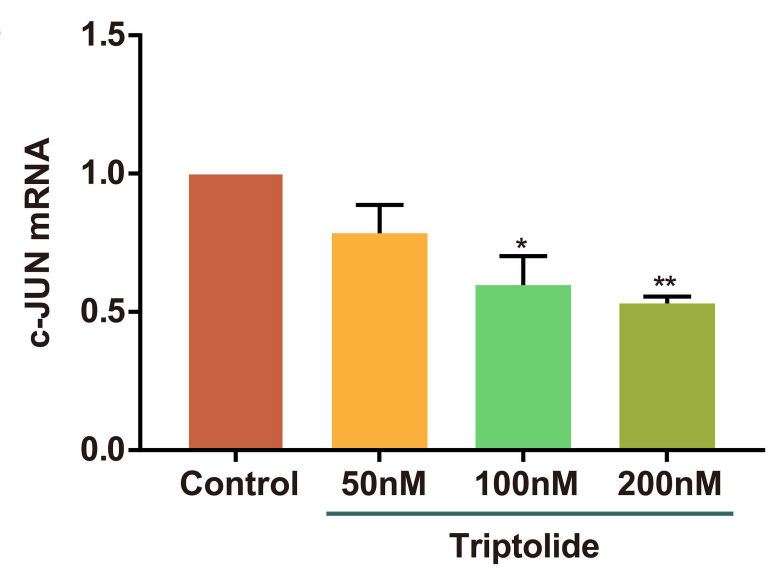

D

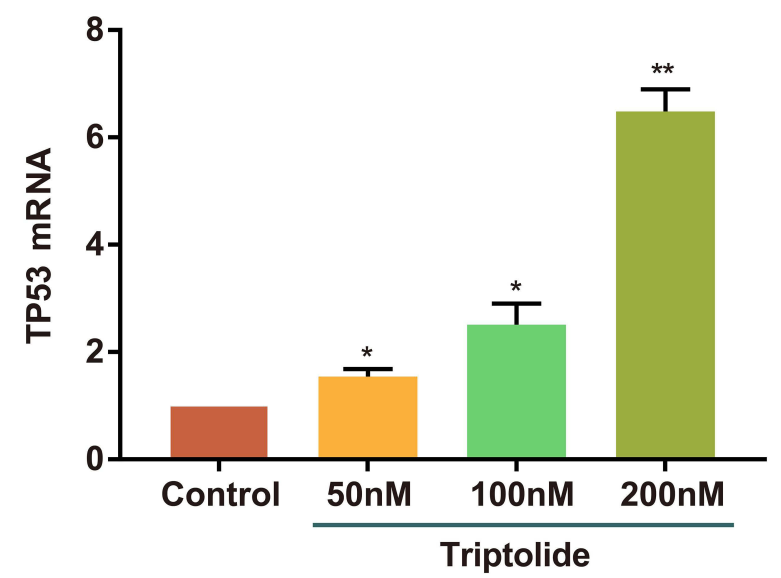

Figure 7 RT-PCR analysis of the regulatory effects of triptolide on the mRNA expression of the core targets. (A) CDKNIA, (B) c-JUN, (C) RELA, (D) TP53. Triptolide regulated the mRNA expression of the core targets in a dose-dependent manner. $* P<0.05, * * P<0.01$. 


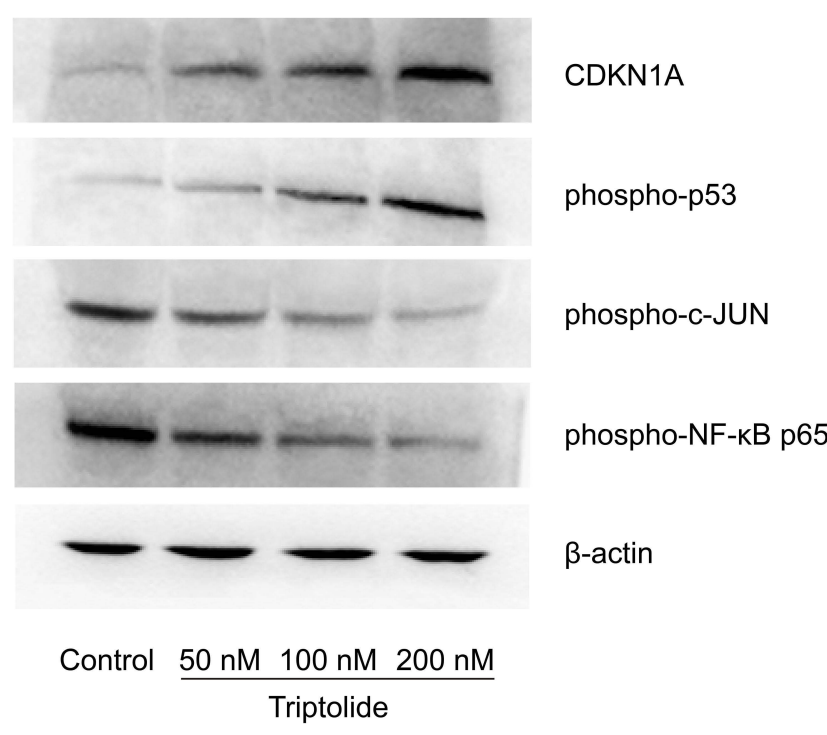

Figure 8 Western blot analysis of the regulatory effects of triptolide on the protein levels of the core targets. CDKNIA, phospho-p53, phospho-c-JUN, phospho-NF $-\kappa B$ p65. Triptolide regulated the protein levels of the core targets in a dosedependent manner (50 nM, $100 \mathrm{nM}, 200 \mathrm{nM})$.

proliferation and induced the apoptosis of thyroid cancer cells. Triptolide could regulate the mRNA expression and protein levels of the core targets in thyroid cancer cells.

In the treatment of thyroid cancer, surgery is initial step in treatment of thyroid cancer in the majority of cases. In more demolitive surgery, interventions are affected by more severe complications. Previous studies have tried to solve this situation. Calò et al used intraoperative neuromonitoring (IONM) in predicting postoperative nerve function during thyroid surgery. ${ }^{10}$ It was highly predictive of postoperative nerve function. Docimo et al found that oral calcium and vitamin D supplements could participate in the prevention of postoperative hypocalcemia. ${ }^{11}$ Conzo et al believed the neck dissection in papillary thyroid cancers management remains controversial which needs to be more investigated. ${ }^{12}$

Triptolide is a kind of diterpenoid epoxide extracted from thunder god vine, Tripterygium wilfordii. ${ }^{13}$ It has been useful in the treatment of various kinds of inflammatory diseases, including rheumatoid arthritis (RA), inflammatory bowel disease (IBD), lupus nephritis, etc. ${ }^{14}$ The functions of triptolide in treating inflammatory diseases are multifactorial. Triptolide can inhibit the production of inflammatory cytokines such as interleukin 1 beta (IL-1 $\beta$ ), interleukin 6 (IL-6), and tumor necrosis factor alpha (TNF- $\alpha$ ). In addition, it can reduce the production of reactive oxygen species to alleviate inflammation. ${ }^{15}$ Triptolide also suppresses the activities of inflammatory cells, including neutrophils, macrophages, dendritic cells, and $T$ cells. In addition, triptolide inhibits signaling related to the NF- $\mathrm{kB}, \mathrm{MAPK}$, and PI3K-Akt signaling pathways. Furthermore, triptolide exhibits anticancer activities. Previous studies have found that triptolide can treat thyroid cancer. Zhu et al demonstrated that triptolide inhibits the invasion and angiogenesis of human anaplastic thyroid carcinoma cells. ${ }^{16} \mathrm{Zhu}$ et al also revealed that triptolide exerts antiangiogenic activity by regulating vascular endothelial and tumor cells. ${ }^{17}$ Although previous studies have revealed that triptolide is useful for treating thyroid cancer, the mechanism underlying this effect has not been thoroughly investigated.

In this study, KEGG analysis showed that the pathways of triptolide in treating thyroid cancer included the cell cycle pathway. GO analysis showed that the biological processes included the terms of cell cycle arrest, G2/M transition of mitotic cell cycle, and G1/S transition of mitotic cell cycle. Therefore, we considered that triptolide could regulate the cell cycle to treat thyroid cancer. The cell cycle, the process by which cells divide and proliferate, is the key mediator of cancer. In normal cells, the cell cycle is controlled by a complex series of signaling pathways through which a cell grows, undergoes DNA replication and divides. ${ }^{18}$ This process also includes mechanisms to ensure that errors are corrected and that cells commit suicide (undergo apoptosis) if they are not corrected. In cancer, this regulatory process malfunctions as a result of genetic mutations and leads to uncontrolled cell proliferation. ${ }^{19}$ Cell cycle progression is accelerated by cyclin-dependent kinases stimulated by cyclins, including cyclin D1, and inactivated by cyclin-dependent kinase inhibitors (CDKIs). ${ }^{20}$ Thus, triptolide could influence the $\mathrm{G} 2 / \mathrm{M}$ and $\mathrm{G} 1 / \mathrm{S}$ transitions in the mitotic cell cycle to regulate the cell cycle in thyroid cancer cells. Furthermore, we found that triptolide could interact with CDKN1A to influence the cell cycle. CDKN1A is implicated in the regulation of cell growth and the cellular response to DNA damage. ${ }^{21}$ It inhibits cell cycle progression in G1 phase by binding to G1 cyclin-CDK complexes and may also induce G2 arrest. $^{22}$ In response to DNA damage, p53 induces CDKN1A expression, which is responsible for cell cycle arrest at the G1 checkpoint. ${ }^{23}$ Our RT-PCR and Western blot results showed that triptolide increased the expression of CDKN1A. Therefore, triptolide could inhibit thyroid cancer proliferation by regulating the cell cycle through binding to CDKN1A. 


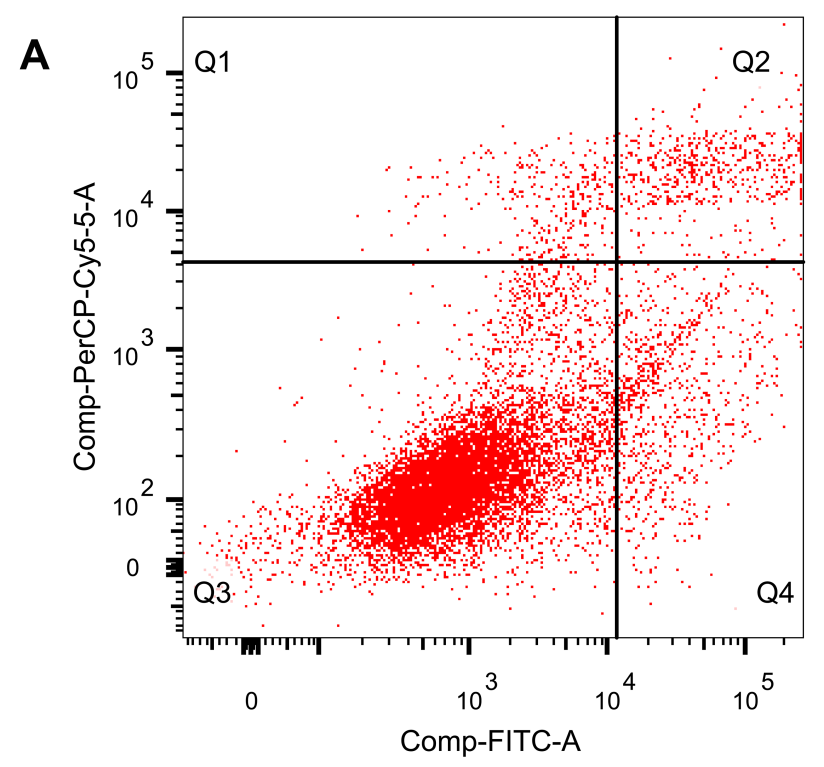

\section{Control}

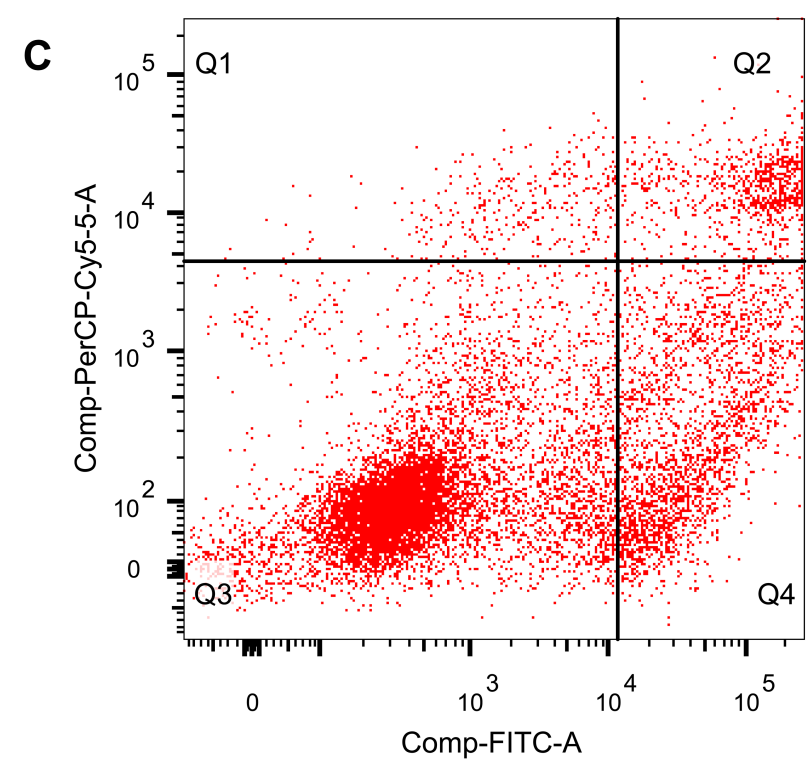

$100 \mathrm{nM}$

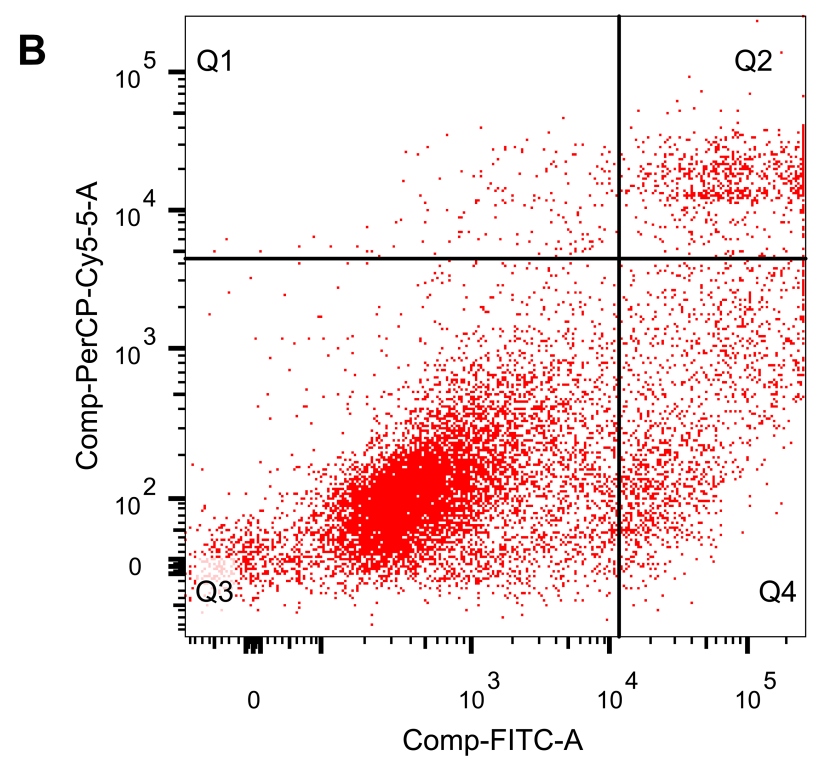

$50 \mathrm{nM}$

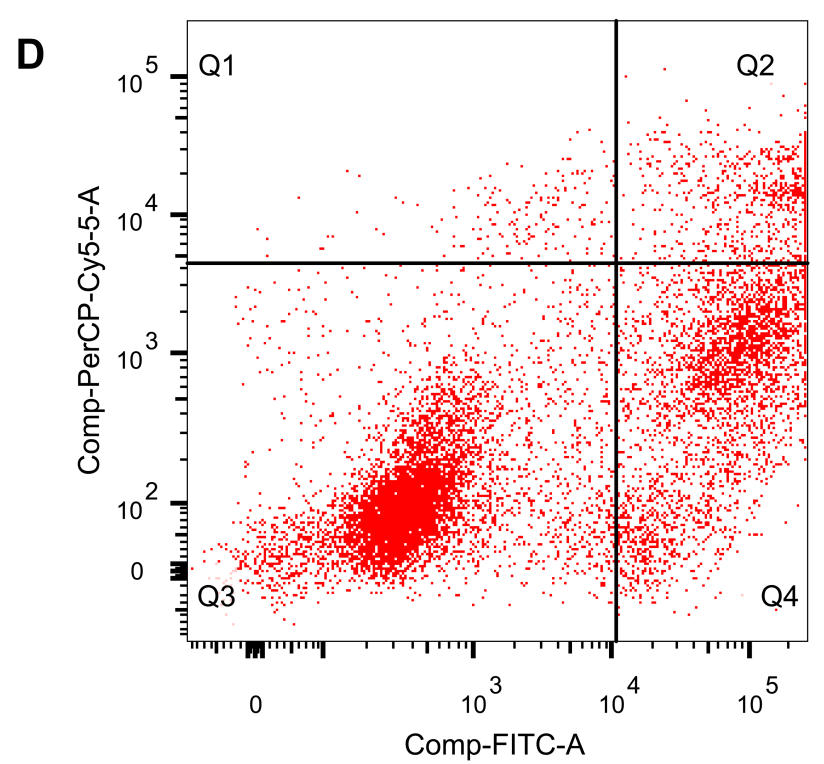

$200 \mathrm{nM}$

Figure 9 Triptolide induced the apoptosis of thyroid cancer cells. A cell apoptosis kit was used to measure the apoptosis of TPC-I cells by flow cytometry. (A) TPC-I cells were incubated with $0 \mathrm{nM}$ triptolide as control. (B) TPC-I cells were incubated with $50 \mathrm{nM}$ triptolide. (C) TPC-I cells were incubated with I00 nM triptolide. (D) TPC-I cells were incubated with $200 \mathrm{nM}$ triptolide. Triptolide induced the apoptosis of TPC-I cells (quadrant Q4; control: 3.4\%, $50 \mathrm{nM:} 7 \%$, 100 nM: 10.9\%, 200 nM: 23.5\%) in a dose-dependent manner.

In addition to the involvement of the cell cycle, KEGG analysis showed that inflammatory pathways were also involved in the antithyroid cancer function of triptolide. The inflammatory pathways included NF-kappa B transcription factor activity, the stress-activated MAPK cascade, and I-kappaB kinase/NF-kappa B signaling. GO analysis also showed that the biological processes were related to NF-kappa B transcription factor activity, the stress-activated MAPK cascade, and I-kappaB kinase/NFkappa B signaling. Thus, the NF- $\mathrm{BB}$ and MAPK signaling pathways were considered to participate in the antithyroid cancer function of triptolide. Inflammation is a characteristic of cancer that plays an important role in its initiation and development. NF- $\mathrm{kB}$ signaling pathway activation can induce the production of inflammatory cytokines, such as TNF- $\alpha$, IL-1, and IL- $6 .{ }^{24}$ These 
inflammatory cytokines can enhance the proliferation and survival of cancer cells. In addition, NF- $\kappa \mathrm{B}$ pathway activation can increase cancer cell invasion, angiogenesis, metastasis, and therapeutic resistance. ${ }^{25}$ In addition to the NF- $\kappa \mathrm{B}$ pathway, the MAPK pathway was also involved in the function of triptolide. The MAPK signaling pathway plays an essential role in regulating gene expression, cell proliferation and the survival of cancer cells. ${ }^{26}$ This pathway regulates oncogenesis, cancer cell growth, and chemotherapeutic resistance. c-JUN may function as a sensor for ERK signaling dynamics. In our study, we discovered that triptolide can directly regulate the expression of c-JUN and NF- $\kappa B$ p65. c-JUN is the regulator of the MAPK pathway, while NF- $\kappa \mathrm{B}$ p65 is the regulator of the $\mathrm{NF}-\kappa \mathrm{B}$ pathway. Therefore, we inferred that triptolide could modulate the NF- $\kappa \mathrm{B}$ and MAPK signaling pathways by interacting directly with c-JUN and NF- $\mathrm{B}$ p 65 .

Finally, we found that triptolide induced the apoptosis of thyroid cancer cells. Apoptosis is characterized by cell membrane blebbing, chromatin condensation, and cell shrinkage. Reduced apoptosis plays an important role in cancer cell development and carcinogenesis. ${ }^{27}$ The mechanisms underlying the reduction in apoptosis include reduced caspase function and an impaired balance of antiapoptotic and proapoptotic proteins. Apoptotic proteins include the Bcl-2 family of proteins, p53 (or TP53), and inhibitor of apoptosis proteins (IAPs). The Bcl-2 family of proteins is a group of proapoptotic and antiapoptotic proteins, such as Bcl-2, B-cell lymphoma-extra large (Bcl-xL) , Mcl-1, and Bcl-w. ${ }^{28}$ Inhibitors of apoptosis proteins, including BIRC1, BIRC2, BIRC3, BIRC4, and BIRC5, can modulate signal transduction, cytokine production, and apoptosis. The P53 protein is a tumor suppressor protein that can induce the apoptosis of cancer cells. ${ }^{29}$ P53 is able to induce apoptosis via transcriptiondependent and transcription-independent mechanisms. Disruption of P53 activates tumor development and progression. P53 can regulate the transcription of $\mathrm{Bcl}-2$ family members. In addition, P53 transactivates some components of the apoptotic effector machinery. ${ }^{30}$ It also induces genes in short-circuit antiapoptotic pathways. In our study, we found that triptolide induced the apoptosis of thyroid cancer cells. Molecular docking analysis showed that triptolide can directly interact with P53. RT-PCR and WB analyses demonstrated that triptolide increased the expression of P53. Thus, we inferred that triptolide could increase the expression of P53 to induce the apoptosis of thyroid cancer cells by influencing the transcription of Bcl-
2 family genes, transactivating some components of the apoptotic effector machinery, and inducing apoptotic pathways. Apart from apoptosis, P53 plays important role in several fundamental cellular signaling pathways including cell cycle arrest. P53 indirectly regulates many important genes expression which are essential in cell division cycle. As the CDK inhibitor, CDKN1A was the transcriptional target of P53. P53 activation could transcriptionally upregulates CDKN1A expression. Then, CDKN1A acts as cell cycle checkpoint control to inhibit cell cycle progression in G1 phase by binding to G1 cyclin-CDK complexes and may also induce G2 arrest. So, P53 might also participate in cell cycle arrest except for apoptosis.

\section{Conclusion}

In conclusion, we discovered the function of triptolide in treating thyroid cancer in this study. Using the network pharmacology method, we found that inflammatory pathways, including the NF- $\mathrm{BB}$ and MAPK signaling pathways, were involved in the antithyroid cancer mechanism of triptolide. In addition, triptolide influenced the cell cycle and apoptosis of thyroid cancer cells. Molecular docking analysis showed that triptolide directly interacted with four core targets: CDKN1A, c-JUN, RELA, and TP53. CDKN1A is related to the cell cycle, while TP53 is related to apoptosis. c-JUN and RELA are related to the $\mathrm{NF}-\kappa \mathrm{B}$ and MAPK signaling pathways. Then, we explored the function of triptolide in vitro. Triptolide inhibited the proliferation and induced the apoptosis of thyroid cancer cells. Triptolide increased the mRNA expression levels of CDKN1A and TP53 but reduced those of c-JUN and RELA in a dose-dependent manner. Triptolide increased the protein levels of CDKN1A and phospho-p53 but reduced those of phospho-c-JUN and phospho-NF-кB p65 in a dose-dependent manner. Therefore, we considered that triptolide could treat thyroid cancer by inhibiting cell proliferation, inducing apoptosis and inhibiting inflammatory pathways such as the NF-кB and MAPK signaling pathways. CDKN1A, c-JUN, RELA, and TP53 were involved in the mechanism of triptolide in the treatment of thyroid cancer.

\section{Abbreviations}

AKAP9, A-Kinase Anchoring Protein 9; BC, betweenness centrality; Bcl-2, B-cell lymphoma 2; Bcl-X1, B-cell lymphoma-extra large; BIND, Biomolecular Interaction Network Database; BioGRID, Biological General 
Repository for Interaction Datasets; BP, biological process; CC, closeness centrality; CC, cellular component; CCKBR, Cholecystokinin B Receptor; CKDN1A, cyclindependent kinase inhibitor 1A; CDKN1B, Cyclin Dependent Kinase Inhibitor 1B; CXCL8, C-X-C Motif Chemokine Ligand 8; DC, degree centrality; DIP, Database for Interacting Proteins; EC, eigenvector centrality; EDTA, Ethylenediaminetetraacetic acid; FBS, fetal bovine serum; FITC, Fluorescein isothiocyanate; GAD, Genetic Association Database; GAPDH, glyceraldehyde 3-phosphate dehydrogenase; GO, Gene ontology; HMGCR, 3-Hydroxy-3-Methylglutaryl-CoA Reductase; HPRD, Human Protein Reference Database; IBD, inflammatory bowel disease; IL-1 $\beta$, interleukin 1 beta; IL-6, interleukin 6; IONM, intraoperative neuromonitoring; JAK1, Janus Kinase 1; KEGG, Kyoto Encyclopedia of Genes and Genomes; LAC, local average connectivity centrality; MAPK, Mitogen-Activated Protein Kinase; MF, molecular function; MINT, Molecular INTeraction Database; NC, neighbourhood centrality; OMIM, Online Mendelian Inheritance in Man; PBST, phosphate buffered saline with Tween 20; PharmGKB, Pharmacogenomics Knowledge Base; PI, propidium iodide; PMSF, phenylmethylsulfonyl fluoride; PPI, protein-protein interaction; RA, rheumatoid arthritis; RT-PCR, real-time polymerase chain reaction; TCMSP, traditional Chinese medicine systems pharmacology; TNF- $\alpha$, tumor necrosis factor alpha; TP53, tumor protein p53; TTD, Therapeutic Target Database.

\section{Acknowledgments}

This work was supported by the National Natural Science Foundation of China [grant numbers 81500689].

\section{Disclosure}

The authors declare that the research was conducted in the absence of any commercial or financial relationships that could be construed as a potential conflict of interest.

\section{References}

1. Cabanillas ME, McFadden DG, Durante C. Thyroid cancer. Lancet. 2016;388(10061):2783-2795. doi:10.1016/S0140-6736(16)30172-6

2. Sung H, Ferlay J, Siegel RL, et al. Global cancer statistics 2020: GLOBOCAN estimates of incidence and mortality worldwide for 36 cancers in 185 countries. CA Cancer J Clin. 2021;71(3):209-249. doi: $10.3322 /$ caac. 21660

3. Asa SL. The current histologic classification of thyroid cancer. Endocrinol Metab Clin North Am. 2019;48(1):1-22. doi:10.1016/j. ecl.2018.10.001
4. Grani G, Lamartina L, Durante C, et al. Follicular thyroid cancer and Hürthle cell carcinoma: challenges in diagnosis, treatment, and clinical management. Lancet Diabetes Endocrinol. 2018;6(6):500-514. doi:10.1016/S2213-8587(17)30325-X

5. Schmidbauer B, Menhart K, Hellwig D, et al. Differentiated thyroid cancer-treatment: state of the art. Int J Mol Sci. 2017;18(6):E1292. doi:10.3390/ijms18061292

6. Noel P, Von Hoff DD, Saluja AK, et al. Triptolide and its derivatives as cancer therapies. Trends Pharmacol Sci. 2019;40(5):327-341. doi:10.1016/j.tips.2019.03.002

7. Jiang W, Chen M, Xiao C, et al. Triptolide suppresses growth of breast cancer by targeting HMGB1 in vitro and in vivo. Biol Pharm Bull. 2019;42(6):892-899. doi:10.1248/bpb.b18-00818

8. Sun YY, Xiao L, Wang D, et al. Triptolide inhibits viability and induces apoptosis in liver cancer cells through activation of the tumor suppressor gene p53. Int $J$ Oncol. 2017;50(3):847-852. doi:10.3892/ijo.2017.3850

9. Reno TA, Kim JY, Raz DJ. Triptolide inhibits lung cancer cell migration, invasion, and metastasis. Ann Thorac Surg. 2015;100 (5):1817-1824. doi:10.1016/j.athoracsur.2015.05.074

10. Calò PG, Medas F, Conzo G, et al. Intraoperative neuromonitoring in thyroid surgery: is the two-staged thyroidectomy justified? Int J Surg. 2017;41(Suppl 1):S13-S20. doi:10.1016/j.ijsu.2017.02.001

11. Docimo G, Tolone S, Ruggiero R, et al. Total thyroidectomy without prophylactic central neck dissection combined with routine oral calcium and vitamin D supplements: is it a good option to achieve a low recurrence rate avoiding hypocalcemia? A retrospective study. Minerva Chir. 2013;68(3):321-328.

12. Conzo G, Docimo G, Mauriello C, et al. The current status of lymph node dissection in the treatment of papillary thyroid cancer. A literature review. Clin Ter. 2013;164(4):e343-e346.

13. Chen SR, Dai Y, Zhao J, et al. A mechanistic overview of triptolide and celastrol, natural products from Tripterygium wilfordii Hook F. Front Pharmacol. 2018;9:104. doi:10.3389/fphar.2018.00104

14. Yuan K, Li X, Lu Q, et al. Application and mechanisms of triptolide in the treatment of inflammatory diseases-a review. Front Pharmacol. 2019;10:1469. doi:10.3389/fphar.2019.01469

15. Hou W, Liu B, Xu H. Triptolide: medicinal chemistry, chemical biology and clinical progress. Eur J Med Chem. 2019;176:378-392. doi:10.1016/j.ejmech.2019.05.032

16. Zhu W, Ou Y, Li Y, et al. A small-molecule triptolide suppresses angiogenesis and invasion of human anaplastic thyroid carcinoma cells via down-regulation of the nuclear factor-kB pathway. Mol Pharmacol. 2009;75(4):812-819. doi:10.1124/mol.108.052605

17. Zhu W, He S, Li Y, et al. Anti-angiogenic activity of triptolide in anaplastic thyroid carcinoma is mediated by targeting vascular endothelial and tumor cells. Vascul Pharmacol. 2010;52(12):46-54. doi:10.1016/j.vph.2009.10.006

18. Calcinotto A, Kohli J, Zagato E, et al. Cellular senescence: aging, cancer, and injury. Physiol Rev. 2019;99(2):1047-1078. doi:10.1152/ physrev.00020.2018

19. Icard P, Fournel L, Wu Z, et al. Interconnection between metabolism and cell cycle in cancer. Trends Biochem Sci. 2019;44(6):490-501. doi:10.1016/j.tibs.2018.12.007

20. Tchakarska G, Sola B. The double dealing of cyclin D1. Cell Cycle. 2020;19(2):163-178. doi:10.1080/15384101.2019.1706903

21. Otto T, Piotr P. Cell cycle proteins as promising targets in cancer therapy. Nat Rev Cancer. 2017;17(2):93-115. doi:10.1038/nrc.20 16.138

22. Hydbring P, Malumbres M, Sicinski P. Non-canonical functions of cell cycle cyclins and cyclin-dependent kinases. Nat Rev Mol Cell Biol. 2016;17(5):280-292. doi:10.1038/nrm.2016.27

23. Malumbres M, Barbacid M. Cell cycle, CDKs and cancer: a changing paradigm. Nat Rev Cancer. 2009;9(3):153-166. doi:10.1038/nrc2602

24. Taniguchi K, Michael M. NF-kB, inflammation, immunity and cancer: coming of age. Nat Rev Immunol. 2018;18(5):309-324. 
25. Eluard B, Thieblemont C, Baud V. NF- $\kappa \mathrm{B}$ in the new era of cancer therapy Trends Cancer. 2020;6(8):677-687. doi:10.1016/j.trecan.2020.04.003

26. Braicu C, Buse M, Busuioc C, et al. A comprehensive review on MAPK: a promising therapeutic target in cancer. Cancers. 2019;11 (10):1618. doi:10.3390/cancers11101618

27. Carneiro BA, El-Deiry WS. Targeting apoptosis in cancer therapy. Nat Rev Clin Oncol. 2020;17(7):395-417.

28. Warren CFA, Wong-Brown MW, Bowden NA. BCL-2 family isoforms in apoptosis and cancer. Cell Death Dis. 2019;10(3):177. doi:10.1038/s41419-019-1407-6
29. Aubrey B, Kelly G, Janic A, et al. How does p53 induce apoptosis and how does this relate to p53-mediated tumour suppression? Cell Death Differ. 2018;25(1):104-113.

30. Hafner A, Bulyk ML, Jambhekar A, et al. The multiple mechanisms that regulate p53 activity and cell fate. Nat Rev Mol Cell Biol. 2019;20(4):199-210. doi:10.1038/s41580-019$0110-\mathrm{x}$

\section{Publish your work in this journal}

Drug Design, Development and Therapy is an international, peerreviewed open-access journal that spans the spectrum of drug design and development through to clinical applications. Clinical outcomes, patient safety, and programs for the development and effective, safe, and sustained use of medicines are a feature of the journal, which has also been accepted for indexing on PubMed Central. The manuscript management system is completely online and includes a very quick and fair peer-review system, which is all easy to use. Visit http://www. dovepress.com/testimonials.php to read real quotes from published authors. 